\title{
POLO: A web interface for the rapid identification of crystals C. Lesburg ${ }^{1}$ \\ ${ }^{1}$ Merck \& Co \\ charles.lesburg@merck.com
}

Advances in protein expression and purification, coupled with innovations in automated liquid handling and imaging, have resulted in efficient broad crystallization screening of macromolecules. In 2018, a consortium of crystallization laboratories and image analysis experts published MARCO, a highly-accurate image classification model (Bruno, 2018). Seeking to apply the benefits of the MARCO model to the day-to-day needs of macromolecular crystallographers, we designed and implemented POLO (Lesburg, 2020), a web-based interface for the rapid identification of crystals. The interface is designed for responsiveness and is focused on the immediate needs of the laboratory scientist. The POLO system is closely integrated with RockMaker (Formulatrix, Bedford, MA) and can support multiple RockMaker databases, multiple classification models, and also provides a streamlined mechanism to store manual annotations for the development of future image classification models. 